\title{
BENDING OF ADSORBED FCN AND FNC MOLECULES INDUCED BY THE RENNER-TELLER EFFECT
}

\author{
Natalia Gorinchoy \\ Institute of Chemistry, Academy of Sciences of Moldova, Academiei str. 3, MD 2028 Chisinau, \\ Republic of Moldova
}

\section{Article Info \\ Received: 11 December 2011 \\ Accepted: 17 January 2012}

Keywords Renner-Teller Effect, adsorption, FCN, FNC.

\begin{abstract}
It is shown that the bending of FCN and FNC molecules adsorbed on Si $(100)-(2 \times 1)$ surface, is due to the Renner-Teller effect induced by the orbital charge transfers by adsorption. From $a b$ initio calculations of free FCN and FNC and the molecules adsorbed on the model $\mathrm{Si}_{9} \mathrm{H}_{12}$ cluster, the orbital charge transfers to and from the molecules were calculated, the vibronic coupling constants were estimated, and the curvature $K$ of the adiabatic potentials for the bending coordinate of adsorbed molecules was evaluated. Calculations show that for both sideon adsorbed species, as well as for end-on adsorbed FNC molecule $K<0$ that leads to their bending. For the end-on adsorbed FCN $K>0$, and this molecule remains linear
\end{abstract}

\section{Introduction}

Numerous experimental and theoretical data indicate that small molecules coordinated to the metal centers or adsorbed on the surfaces undergo, as a rule, structural changes leading to distortion of their high-symmetry nuclear configurations. Sufficient to mention the distortion of the linear configuration of free acetylene molecule to the non-linear cis-form by its $\eta^{2}$-coordination to the metal centers [1-5], the out-of-plane distortion of coordinated ethylene molecule [6-11], the changes in white phosphorus $\left(\mathrm{P}_{4}\right)$ geometry upon coordination in transition metal complexes [12-15], the bending of coordinated or adsorbed $\mathrm{CO}_{2}$ molecule [16-18], etc.

In our recent work [19] a new approach to handle structural changes in coordinated molecules was worked out based on an approximate evaluation of the Jahn-Teller effect (JTE), pseudo JTE (PJTE), and Renner-Teller effect (RTE) in such systems. The structural changes in such subsystems can be described by means of small orbital charge transfers (OCT) $\Delta q<<n e$, where $n$ is the number of electrons in the system, which can be considered as 
small perturbations to the integer-electron system. The theory involves orbital vibronic coupling constants and orbital force constants [20], and estimates the vibronic effects in the approximation of molecular orbital theory. Some applications of the theory to specific coordination compounds demonstrated that in all the cases distortions of coordinated molecules are due to the JTE, PJTE or RTE induced by the OCTs to and from molecular orbitals of coordinated ligands [19]. In [21] the adsorption of FCN and FNC molecules on Si (100)-(2×1) surface has been studied by DFT B3LYP method with the $\mathrm{Si}_{9} \mathrm{H}_{12}$ cluster model. Among other things, it was shown that both side-on adsorbed molecules, as well as the endon adsorbed FNC are bent, whereas the end-on adsorbed FCN remains linear. However, the reasons for the bent structure of the adsorbed molecules have not been clarified.

In the present paper, we employ the above approach [19] to explain the origin of bending of FCN and FNC molecules adsorbed on Si $(100)-(2 \times 1)$ surface as due to the Renner-Teller effect induced by the OCTs by adsorption.

\section{Theoretical model and calculation details}

The idea of the method [19] is that the influence of a small orbital charge transfers (OCT) $\Delta q$ can be considered as a small perturbation to the integer-electron system. In the molecular orbital (MO) approximation the additional charge occupies the LUMO or frees the HOMO without changing significantly the MO wavefunction in the first order perturbation theory. As mentioned in the introduction, the theory involves the concept of orbital vibronic coupling constants (OVCC) introduced earlier [20, p. 340]:

$$
f_{Q}^{(i, j)}=\left\langle i\left|(\partial H / \partial Q)_{0}\right| j\right\rangle
$$

where $|i\rangle$ and $|j\rangle$ are the MO wavefunctions, $Q$ is the symmetrized coordinate of nuclear displacements, and $H$ is the Hamiltonian. The diagonal OVCC $f_{Q}^{(i, i)} \equiv f_{Q}^{i}$ has the physical meaning of the force with which the electron on the $i$-th MO distorts the nuclear framework in the direction of $Q$, and in the approximation under consideration the total distortion force, the integral vibronic coupling constant $F$, equals the sum of OVCC multiplied by the MO occupation numbers $q_{\mathrm{i}}$ [20],

$$
F_{Q}=\sum_{i} q_{i} f_{Q}^{i}
$$


Assume that before the charge transfer the system is stable in a given nuclear configuration $Q_{0}=0$, meaning that in this configuration $F_{Q}^{\prime}=\sum_{i} q_{i}^{\prime} f_{Q}^{i}=0$. Then after the charge transfers $\Delta q_{i}$ this force may become non-zero,

$$
F_{Q}=F_{Q}^{\prime}+\Delta F=\sum_{i}\left(q_{i}^{\prime}+\Delta q_{i}\right) f_{Q}^{i}=\sum_{i} \Delta q_{i} f_{Q}^{i}
$$

This equation, in principle, solves the problem of the JTE in systems with fractional charges. Indeed, consider the case when the system is stable in the high-symmetry configuration and only one MO takes part in the charge transfer with the additional charge $\Delta q_{i}$ occupying a degenerate orbital. For this latter, in accordance with the JTE, $f_{Q}^{i} \neq 0$ in the direction of JT active coordinates $Q$. Acting upon the nuclear framework, this non-zero force $\Delta q_{i} f_{Q}^{i}$ distorts it in the direction $Q$. A similar JTE emerges when the charge $\Delta q_{i}$ is removed from a fully occupied degenerate orbital (which does not produce distortions without the charge transfer).

Similar structural changes take place in the Renner-Teller (RT) situation, with the exception that the vibronic coupling constants between the two components of the degenerate state in this case vanish for linear nuclear displacements, so the nonzero effect takes place only for quadratic coupling [20] with the vibronic coupling constant

$$
g_{Q}^{(i, j)}=\left\langle i\left|\left(\partial^{2} H / \partial Q^{2}\right)_{0}\right| j\right\rangle
$$

where $|i\rangle$ and $|j\rangle$ are the two MOs of the degenerate $\Pi$ state. In this case the RT effect splits the energy term $E(Q)$, but the components remain quadratic in the nuclear displacements $Q$; for a double degenerate term

$$
E_{ \pm}=(1 / 2)\left(K_{0} \pm g_{Q}^{(i, j)}\right) Q^{2}
$$

If both orbitals forming the double degenerate term are equally populated the total effect is zero (in such case the term is nondegenerate). In the frozen orbital approximation under consideration the orbital populations can be taken into account by the substitution $g_{Q}^{(i, j)} \rightarrow\left(q_{i}-q_{j}\right) g_{Q}^{(i, j)}$, and the charge transfers $\Delta q_{\mathrm{i}}$ and $\Delta q_{\mathrm{j}}$ change the RTE accordingly,

$$
E_{ \pm}=(1 / 2)\left[K^{\text {free }} \pm\left(q_{i}-q_{j}\right) g_{Q}^{(i, j)}\right] Q^{2}
$$

Thus, in order to clarify whether the adsorbed linear molecule is stable $\left(K^{\text {adsorb }}>0\right)$ or unstable $\left(K^{\text {adsorb }}<0\right)$ with respect to bending, we need to estimate the curvature $K^{\text {adsorb }}$ of the AP of this molecule in the $\pi$ direction (bending mode), 


$$
K^{\text {adsorb }}=K^{\text {free }}-\left(q_{i}-q_{j}\right) g_{Q}^{(i, j)}
$$

For this it is necessary to calculate the curvature of the AP of free molecule $\left(K^{\text {free }}\right)$, to estimate the RT coupling constants $g_{\pi}^{(i, j)}$, and to evaluate the orbital charge transfers induced by adsorption. To do this the electronic structure of free FCN and FNC molecules and the molecules adsorbed on Si (100)-(2×1) surface was evaluated.

As in [21], a single-dimer $\mathrm{Si}_{9} \mathrm{H}_{12}$ model cluster was used to represent the reconstructed Si (100)-(2×1) surface. The geometry optimization of all the systems was carried out in the frame of the RHF method using the $6-31 \mathrm{G}$ basis set. The potential energy curves for free FCN and FNC molecules and for their positive and negative ions as the functions of the bending modes were calculated taking into consideration configuration interaction (CI). The CI matrices included all electron configurations produced by single and double excitations from three highest occupied to the lowest three unoccupied MOs. All calculations were performed with the GAMESS quantum chemistry package [22].

To estimate the values of the orbital charge transfers to and from adsorbed molecules, the calculated MOs of the entire systems (adsorbed molecule - $\mathrm{Si}_{9} \mathrm{H}_{12}$ cluster) are rewritten in the basis of the eigenfunctions of the free molecules and the atomic orbitals of other atoms. Then the changes in the occupations of MOs of adsorbed molecules are estimated from the difference in Mulliken populations of the corresponding orbitals.

\section{Results and Discussions}

\subsection{Geometries of free and adsorbed FCN and FNC molecules}

At the first stage of our investigation the equilibrium geometries and electronic structure of FCN and FNC molecules were calculated. Both neutral isomers show linear geometries and closed-shell ${ }^{1} \Sigma^{+}$electronic ground state with the valence-shell electronic configurations $\quad(1 \sigma)^{2}(2 \sigma)^{2}(3 \sigma)^{2}(1 \pi)^{4}(4 \sigma)^{2}(2 \pi)^{4}(5 \sigma)^{0}(3 \pi)^{0} \quad$ and $(1 \sigma)^{2}(2 \sigma)^{2}(3 \sigma)^{2}(1 \pi)^{4}(2 \pi)^{4}(4 \sigma)^{2}(3 \pi)^{0}(5 \sigma)^{0}$ for FCN and FNC molecules respectively.

The calculated geometrical parameters and vibrational frequencies for free molecules are listed in Table 1 together with those obtained by other authors and with the available experimental data. It is seen that our results agree well with the DFT-B3LYP values given in Ref. [21] and with the experimental data [23-25]. This indicates that the present method is able to describe correctly both FCN and FNC systems. 
Table 1. Bond lengths $R_{\mathrm{A}-\mathrm{B}}(\AA)$ and vibrational frequencies $\left(\mathrm{cm}^{-1}\right)$ for free FCN and FNC molecules

\begin{tabular}{|c|c|c|c|c|c|c|}
\hline & \multicolumn{3}{|c|}{$\mathrm{FCN}$} & \multicolumn{3}{|c|}{ FNC } \\
\hline & $\begin{array}{l}\text { This } \\
\text { work }\end{array}$ & [21] & Exp. & $\begin{array}{l}\text { This } \\
\text { work }\end{array}$ & [21] & Exp. \\
\hline$R_{\mathrm{C}-\mathrm{N}}$ & 1.14 & 1.15 & $1.16^{\mathrm{a})}$ & 1.17 & 1.18 & \\
\hline$R_{\mathrm{F}-\mathrm{C}}$ & 1.28 & 1.26 & $1.26^{\mathrm{a})}$ & & & \\
\hline$R_{\mathrm{F}-\mathrm{N}}$ & & & & 1.32 & 1.30 & \\
\hline$v_{\mathrm{C}-\mathrm{N}}$ & 2411 & 2343 & $2319^{b)}$ & 2208 & 2155 & $2123^{c)}$ \\
\hline$v_{\mathrm{C}-\mathrm{F}}$ & 1106 & 1074 & $1076^{b)}$ & & & \\
\hline$v_{\mathrm{N}-\mathrm{F}}$ & & & & 994 & 961 & $928^{c)}$ \\
\hline$v_{\mathrm{FCN}}$ & 468 & 496 & $451^{b)}$ & & & \\
\hline$v_{\mathrm{FNC}}$ & & & & 242 & 267 & \\
\hline
\end{tabular}

Fig.1 shows the optimizes structures of the FCN and FNC molecules adsorbed on the $\mathrm{Si}_{9} \mathrm{H}_{12}$ cluster in both end-on and side-on adsorption modes. Calculated geometry parameters of adsorbed FCN and FNC molecules are presented in Table 2.

\section{Side-on}
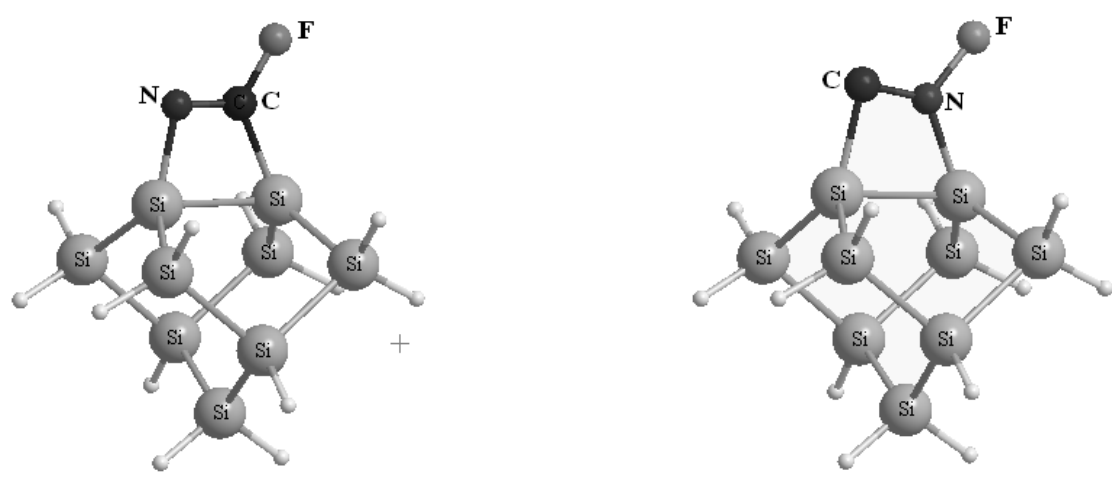

End-on
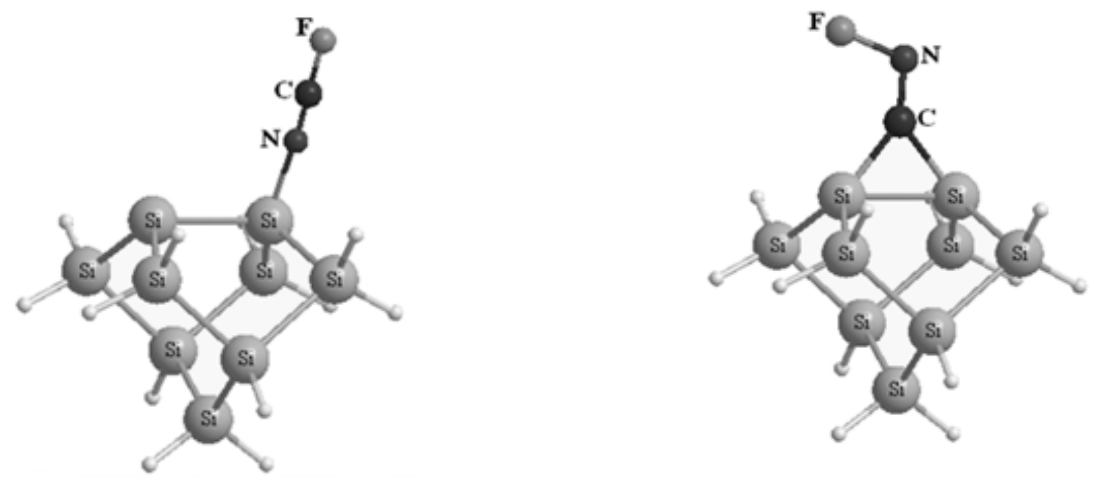

Fig 1. Optimized structures of adsorbed FCN and FNC molecules 
Table 2. Geometry parameters of adsorbed FCN and FNC molecules (bond lengths in $\AA$, bond angles in degrees). In parentheses are data from Ref. [21]

\begin{tabular}{lccccc}
\hline & \multicolumn{2}{c}{$\mathrm{FCN}$} & & \multicolumn{2}{c}{$\mathrm{FNC}$} \\
\cline { 2 - 3 } \cline { 5 - 6 } & End-on & Side-on & & End-on & Side-on \\
\hline$R_{\mathrm{C}-\mathrm{N}}$ & $1.15(1.15)$ & $1.26(1.27)$ & & $1.25(1.26)$ & $1.30(1.27)$ \\
$R_{\mathrm{F}-\mathrm{C}}$ & $1.27(1.25)$ & $1.37(1.33)$ & & & \\
$R_{\mathrm{F}-\mathrm{N}}$ & & & & $1.46(1.42)$ & $1.43(1.49)$ \\
$\angle \mathrm{FCN}$ & $179.7(180.0)$ & $121.8(121.0)$ & & & \\
$\angle \mathrm{FNC}$ & & & & $109.1(110.0)$ & $118.2(119.5)$ \\
\hline
\end{tabular}

As seen from Table 1 and Fig. 1, the adsorbed molecules undergo considerable structural modifications: the bond lengths change significantly compared with those for free molecules, and in the three cases the molecules are bent.

\subsection{Orbital charge transfers and Renner-Teller effect in adsorbed FCN and FNC molecules}

From Section 2 it follows that in order to clarify whether the adsorbed molecule is stable or unstable with respect to bending, we need to estimate the curvature $K$ (Eq. 7 ) of the AP of this molecule in the $\pi$ direction (bending mode). The normal modes of the bending distortion, $Q_{x}$, are:

$$
\begin{aligned}
& Q_{x}(F C N)=-0.0737 x_{F}+0.2458 x_{C}-0.1106 x_{N} \\
& Q_{x}(F N C)=-0.0754 x_{F}+0.2188 x_{N}-0.1359 x_{C}
\end{aligned} .
$$

Calculating the total energies $E\left(Q_{x}\right)$ of free FCN and FNC molecules as the functions of the normal coordinates (Eq. 8) at small values of $Q_{x}$ we find $K_{\pi}^{\text {free }}(\mathrm{FCN})=0.47 \mathrm{ev} / \AA^{2}$ and $K_{\pi}^{\text {free }}(\mathrm{FNC})=0.28 \mathrm{ev} / \AA^{2}$.

When molecules are adsorbed on the surface, two OCTs can take place: a charge transfer from the HOMO ( $2 \pi$ for FCN or $4 \sigma$ for FNC) to the cluster, $\Delta q(\mathrm{HOMO})<0$, and from the cluster to the unoccupied degenerate $3 \pi \mathrm{MO}$ of molecules, $\Delta q(3 \pi)>0$. Note first of all that removal of some part of electron density from the HOMOs does not change significantly the curvature of the AP of considered molecules to bending. The arguments for this conclusion are the following.

Consider the limiting case of the cations $\mathrm{FCN}^{+}$and $\mathrm{FNC}^{+}$. Removal of an electron from the highest occupied $2 \pi \mathrm{MO}$ of FCN molecule gives rise to the ground electronic state of the corresponding cation, i.e., the ${ }^{2} \Pi$ state. The geometry of the ${ }^{2} \Pi$ state of $\mathrm{FCN}^{+}$is calculated to be linear with decreased FC and increased $\mathrm{CN}$ bond distances, reflecting the bonding and antibonding characters of the $2 \pi$ orbital with respect to the FC and $\mathrm{CN}$ bonds, respectively. Ionization of FCN leads to an insignificant reduction of the curvature by the value of 0.05 
ev $/ \AA^{2}$, calculated $K_{\pi}\left(\mathrm{FCN}^{+}\right)=0.42 \mathrm{ev} / \AA^{2}$. This small value $0.05 \mathrm{ev} / \AA^{2}$ can be attributed to the quadratic coupling constant $g(2 \pi)$. This result agrees well with the data from the paper [26], in which the vibrational frequency of the bending vibration of the ground ${ }^{2} \Pi$ state of $\mathrm{FCN}^{+}$of only $\approx 60 \mathrm{~cm}^{-1}$ less than that in the neutral molecule, and the dimensionless RT parameter $\epsilon$ is equal to 0.2485 , which corresponds to a rather weak RT effect. As for the FNC molecule, then its HOMO $4 \sigma$ is mainly the lone pair of carbon atom. Removal of an electron from this MO does not change the curvature to bending.

Thus, the bending of the adsorbed molecules can only be a consequence of the RT effect induced by the charge transfer to the unoccupied $3 \pi$ MO. From analysis of molecular orbitals of entire systems (adsorbed molecule $-\mathrm{Si}_{9} \mathrm{H}_{12}$ cluster), the following values of the orbital charge transfers are obtained: for molecules adsorbed with a side-on position $\Delta q(3 \pi)=$ $0.78 \mathrm{e}$ for FCN and $\Delta q(3 \pi)=0.80 \mathrm{e}$ for FNC; with the end-on mode of adsorption $\Delta q(4 \sigma)=-$ $0.11 \mathrm{e}$ and $\Delta q(3 \pi)=0.82 \mathrm{e}$ for FCN and FNC respectively. Then, calculating the values of splitting between the two components of the ${ }^{2} \Pi$ terms as the functions of the bending coordinates for the anions $\mathrm{FCN}^{-}$and $\mathrm{FNC}^{-}$and using Eq. 5, we obtain the following estimates for parameters $g(3 \pi): g(3 \pi)=1.43 \mathrm{eV} / \AA^{2}$ and $g(3 \pi)=1.24 \mathrm{eV} / \AA^{2}$ respectively for $\mathrm{FCN}$ and FNC.

With these numerical data we get for the negative contribution of the RTE $\Delta K$ to the curvature $K$ of the APES of the free FCN and FNC molecules (in the $\pi$ direction) due to their adsorption: for FNC $\Delta K($ side-on $)=0.99 \mathrm{eV} / \AA^{2}$ and $\Delta K($ end-on $)=1.02 \mathrm{eV} / \AA^{2}$, for FCN $\Delta K($ side-on $)=1.12 \mathrm{eV} / \AA^{2}$ and $\Delta K($ end-on $)=0.006 \mathrm{eV} / \AA^{2}$. Hence the resulting values of the curvature are: $\Delta K($ side-on $)=-0.71 \mathrm{eV} / \AA^{2}, \Delta K($ end-on $)=-0.74 \mathrm{eV} / \AA^{2}$ for $\mathrm{FNC}$, and $\Delta K($ sideon $)=-0.65 \mathrm{eV} / \AA^{2}$ and $\Delta K($ end-on $)=0.464 \mathrm{eV} / \AA^{2}$ for FCN. It is seen that except the end-on adsorbed FCN molecule, the curvature in the $\pi$ direction, $K-\Delta K$, becomes negative in the other three cases. This explains the origin of the bent geometry of adsorption of these molecules and provides for numerical estimates of the strength of the distortion. The larger the absolute value of $K$, the greater the distortion of the molecule (See Table 2).

In the same approximation, by applying the above equations to the adsorbed molecules with respect to the totally symmetric displacements $Q$ of $\Sigma^{+}$symmetry, corresponding to the $\mathrm{C}$-N stretching mode of free molecules (all atoms are situated along the $z$-axis):

$$
\begin{aligned}
& Q_{\Sigma^{+}}(F C N)=-0.032 z_{F}+0.231 z_{C}-0.156 z_{N} \\
& Q_{\Sigma^{+}}(F N C)=-0.034 z_{F}+0.203 z_{N}-0.183 z_{C}
\end{aligned}
$$


we can explain also the alteration in the $\mathrm{C}-\mathrm{N}$ bond lengths upon adsorption with different positions. For the end-on adsorbed FCN molecule, as discussed earlier, only $0.11 \mathrm{e}$ leaves the bonding (with respect to $\mathrm{C}-\mathrm{N}$ bonding) $2 \pi-\mathrm{MO}$. The distorting force $F_{\Sigma}$ in this case changes by the value $\Delta F_{\Sigma}=\Delta q(2 \pi) f_{\Sigma}^{2 \pi}, \Delta q(2 \pi)<0$. It was shown in [20] that for bonding $\operatorname{MO} f^{(i)}>0$, and for antibonding MO $f^{(i)}<0$. Hence $f_{\Sigma}^{2 \pi}>0$ and $\Delta F_{\Sigma}<0$, and we observe a slight stretching of the $\mathrm{C}-\mathrm{N}$ bond. At the same time, this $2 \pi-\mathrm{MO}$ is the antibonding with respect to $\mathrm{C}-\mathrm{F}$ bonding, so that the extraction of charge from this MO results in some contraction of C-F bond.

In the other three cases (both side-on adsorbed molecules and end-on adsorbed FNC) the charge transfer on the antibonding (with respect to $\mathrm{C}-\mathrm{N}$ bonding) $3 \pi^{*}$-MO takes place. Thus, for them $F_{\Sigma}$ changes by $\Delta F_{\Sigma}=\Delta q\left(3 \pi^{*}\right) f_{\Sigma}^{3 \pi^{*}}$. Given that $f_{\Sigma}^{3 \pi^{*}}<0$, and $\Delta q\left(3 \pi^{*}\right)>0$, we get $\Delta F_{\Sigma}<0$. Thus, this charge transfer induces a distorting force which pushes away the carbon and nitrogen nuclei and thereby increases the $\mathrm{C}-\mathrm{N}$ bond length, the distorting force being dependent on the OCT values.

\section{Conclusions}

Our results demonstrate the efficiency of proposed earlier approach [19] in rationalization of the experimental and theoretical data on structural changes in molecular systems due to their interaction with another system. It is shown that bending of FCN and FNC molecules adsorbed on Si (100) surface can be explained as due to the Renner-Teller effect induced by the orbital charge transfers by adsorption. Calculations show that except for the end-on adsorbed FCN molecule, the curvature of the adiabatic potential in the $\pi$ direction becomes negative for both side-on adsorbed molecules and for the end-on adsorbed FNC, which leads to bending of these molecules.

\section{References}

[1] R. S. Walters, P. R. Schleyer, C. Corminboeuf, and M.A. Duncan, J. Amer. Chem. Soc., 127 (2005) 1100

[2] A. Reisinger et al., Angew. Chem. Int Ed., 46 (2007) 8295

[3] A. Krapp, G. Frenking, Angew. Chem. Int. Ed., 47 (2008) 7796

[4] D. Himmel et al., Angew. Chem. Int. Ed., 47 (2008) 7798

[5] J. Datka, E. Kukulska-Zając and W. Kobyzewa, Catalysis Today, 101 (2005) 123

[6] W. Scherer et al., New J. Chem., 30 (2006) 309

[7] N. Hebben et al., Chem. Eur. J., 13 (2007) 10078 
[8] C. Karunatilaka, B. S. Tackett, J. Washington, and S. G. Kukolich J. Am. Chem. Soc., 129 (2007) 10522

[9] A. Dedieu, Chem. Rev., 100 (2000) 543

[10] A. V. Makarov, O. P. Charkin, N. M. Klimenko, Russian J. Inorg. Chem., 55 (2010) 229

[11] J. Oxgaard, G. Bhalla, R. A. Periana, W. A. Goddard III, Organometallics, 25 (2006) 1618

[12] W. W. Schoeller, Inorg. Chem., 50 (2011) 22

[13]. O. J. Scherer, Angew. Chem. Int. Ed., 29 (1990) 1104

[14]. M. Peruzzini, L. Gonsalvia, A. Romerosa, Chem. Soc. Rev., 34 (2005) 1038

[15]. P. Barbaro, et al., Eur. J. Inorg. Chem., (2008) 1392

[16]. X. Yin, J. R. Moss, Coord. Chem. Rev., 181 (1999) 27

[17]. F. Rondinelli, N. Russo, M. Toscano, Theor. Chem. Acc., 115 (2006) 434

[18]. R. Srivastava, T. H. Bennur, D. Srinivas, J. Mol. Catalysis A: Chemical, 226 (2005) 199

[19] N. N. Gorinchoy, I. I. Balan, I. B. Bersuker, Comput.\&Theoret. Chem., 976, (2011) 113

[20] I. B. Bersuker, Electronic Structure and Properties of Transition Metal Compounds. Introduction to the Theory, Second Ed., Wiley, New York (2010)

[21] J. M. Hu, Y. Li, Y. F. Zhang, J. Q. Li, Y. Chen, J. Mol. Struct. (THEOCHEM), 724 (2005) 25

[22] Alex A. Granovsky, www http://classic.chem.msu.su/gran/gamess/index.html; M. W. Schmidt et.al., J. Comput. Chem., 14 (1993) 1347

[23] C. D.i Esposti, P. G. Favero, S. Serenellini, G. Cazzoli, J. Mol. Struct., 82 (1982) 221

[24] V. K. Wang, J. Overend, Spectrochim. Acta, 29a (1973) 1623

[25] D. E. Milligan, M. E. Jacox, J. Chem. Phys., 47 (1967) 278

[26] S. Mishra, V. Vallet, L. V. Poluyanov, W. Domcke, J. Chem. Phys., 123 (2005) 124104 